\title{
Guest Editorial: New Trends in Sliding Mode Control and Observation for Markovian Jump Systems
}

Markovin jump systems (MJSs) are widely utilized to model many dynamical systems with abrupt structural variations governed by a Markov chain. MJSs have been successfully used in many practical systems such as manufacturing processes, economic systems, networked control systems. Due to the theoretical and practical challenges in analysis and synthesis of MJSs, in the past few decades, development of robust control or observer methodologies for this kind of systems has been a main core of theoretical and practical research in control systems community. Specifically, the complexities of MJSs include, but are not limited to, abrupt changes in their structures or parameters and incomplete transition probabilities (TPs). The complexity of the system causes considerable challenges in the tasks of analyzing stability and designing corresponding controller /observer. Therefore, there are two important subjects in system and control theory, which have been extensively studied in the past years. Specially, the sliding mode control and observation $(\mathrm{SMC} / \mathrm{O})$ techniques serve as powerful tools in this respect. Furthermore, some combined SMC/O methodologies have been developed such as adaptive SMC/O, fuzzy-model-based SMC/O and backstepping-based SMC/O for a wide range of MJSs. Therefore, it is still interesting and challenging to develop novel theories of SMC/O for MJSs with possibility of implantation to modern practical application potentials.

The primary objective of this Special Issue is to provide a forum for researchers and practitioners to exchange their latest theoretical and technological achievements and to identify critical issues and challenges for future investigation on the sliding mode control and observation techniques for MJSs. With a stringent peer review process, there are 18 papers accepted by this special issue. Among the accepted papers, nine papers are dedicated to SMC for ccomplex MJSs, nine papers are dedicated to various industrial applications. A summary of the accepted papers is discussed as follows:

\section{SMC for complex MJSs}

For MJSs, the ideal knowledge on the transition probabilities are definitely expected to simplify the system analysis and design. However, the likelihood of obtaining such available knowledge is actually questionable, and the cost is probably expensive. In the paper entitled "Robust Observer Based Fault-tolerant Control for One-sided Lipschitz Markovian Jump Systems with General Uncertain Transition Rates", the authors developed an observer based fault-tolerant sliding mode controller for the one-sided Lipschitz MJSs where the TPs are uncertain.

Compared with the traditional time-triggered mechanisms, the event-triggered transmission schemes can efficiently reduce the communication burden. The authors in the paper with the title "Observer-based Event-triggered Sliding Mode Control for Markov Jump Systems with Partially Unknown Transition Probabilities" designed an observer-based eventtriggered sliding mode controller for MJSs with partially unknown TPs.

In the work named "Sliding Mode Control for a Class of Nonlinear Positive Markov Jumping Systems with Uncertainties in a Finite-time Interval" developed a finite-time sliding mode controller for a class of nonlinear positive MJSs with parameter uncertainties.

In practice, the actuator saturation/faults is inevitable due to the limited capacity. In the paper entitled "Time-varying Barrier Lyapunov Function Based Adaptive Neural Controller Design for Nonlinear Pure-feedback Systems with Unknown Hysteresis", the authors designed an adaptive neural sliding mode controller for nonlinear MJSs with input saturation.

Another work entitled "Fuzzy Adaptive Finite Time Fault-tolerant Control for Multi-input and Multi-output Nonlinear Systems with Actuator Faults" considered the actuator faults of the nonlinear systems and proposed a fuzzy adaptive faulttolerant SMC method.

Time delay occurs widely in practical systems such as biological systems, networked systems and so on, often as a source of instability. In the paper entitled "Sliding Mode Observer Design for Discrete Nonlinear Time-delay Systems with Stochastic Communication Protocol", the authors designed a sliding mode observer for discrete nonlinear time-delay 
systems under the stochastic communication protocol modeled by MJSs.

Another work on the time delay MJSs is named "Sliding Mode Control for Fuzzy Markovian Jump Singular System with Time-varying Delay". Different from the previous work, in this paper, a novel sliding mode controller was developed for fuzzy singular MJSs with time-varying delay.

MJSs are usually utilized to model the random Dos attack in a networked system. In the paper entitled "Robust stochastic sampled-data-based output consensus of heterogeneous multi-agent systems subject to random DoS attack: A Markovian jumping system approach", the authors modeled the random Dos attach by a MJS model, and studied the SMC problem for heterogeneous multi-agent systems.

T-S fuzzy model is well known for its convenience and flexibility in handling complex nonlinear systems. The authors in "Fuzzy Dynamic Sliding Mode Controller Design for Uncertain Nonlinear Markovian Jump Systems" investigated the SMC problem for T-S fuzzy model-based nonlinear MJSs.

\section{Industrial Applications}

By using a new hydraulic transformer, the authors in "Adaptive Fuzzy Sliding Mode Control Based on Pi-sigma Fuzzy Neutral Network for Hydraulic Hybrid Control System Using New Hydraulic Transformer" proposed an adaptive fuzzy sliding mode controller for a hydraulic hybrid control model modeled by MJS.

The authors in the paper entitled "Data-driven Sliding Mode Control for MIMO Systems and Its Application on Linear Induction Motors" presented a data-driven SMC design method for MJSs, and was successfully applied to linear induction motors.

SMC is an efficient way to deal with underactuated systems. The authors of the paper entitled "Spatial Path Following Control of an Autonomous Underactuated Airship" employed the MJS to model the underactuated airship and studied its SMC problem.

The authors in the paper with the title "Enhance Transparency of Force Feedback Interaction Series Mechanism by SMC Strategy" adopted Makov jump model to enhance the transparency of force feedback interaction series mechanism, and proposed a SMC strategy.

Active suspension system plays an important role in supporting the vehicle weight, providing effective isolation of the chassis from road excitations, keeping tire contact with the ground, and maintaining the wheels in appropriate position on the road surface. In the paper entitled "Fault-tolerant Controller Design for Active Suspension System with Proportional Differential Sliding Mode Observer", based on proportional differential sliding mode observer, the authors designed a fault-tolerant controller for active suspension systems.

The authors in the paper with the title "The Equivalent Sliding Mode Tension Control of Carbon Fiber Multilayer Diagonal Loom" developed a sliding mode tension controller for the carbon fiber multilayer diagonal loom.

In order to improve the attitude control performance for synthetic aperture radar satellites, the authors in Coordinated Attitude Control for Synthetic Aperture Radar Satellites with Quantization and Communication Delay" provided a SMC-based coordinated attitude control strategy for synthetic aperture radar satellites with quantization and communication delay.

Another work on satellites is named "Huber Second-order Variable Structure Predictive Filter for Satellites Attitude Estimation". Different from the previous work, in this paper, a huber second-order sliding mode predictive filter was proposed to improve the attitude estimation performance.

The authors in the paper entitled "Sliding Mode Control for We-energy Based on Markovian Jumping Systems" modeled the we-energy model as a Makovian jumping system, and presented a suitable SMC design strategy. 


\section{Acknowledgments}

We really appreciate all the authors and anonymous reviewers who contributed to this Special Issue. Meanwhile, we would like to thank the supports from the Editor-in-Chief of IJCAS, Prof. Keum-Shik Hong to our special issue.

\section{Guest Editors}

Hamid Reza Karimi

Department of Mechanical Engineering

Politecnico di Milano, 20156 Milan, Italy

e-mail: hamidreza.karimi@polimi.it

\section{Qingbin Gao}

School of Mechanical Engineering and Automation Harbin Institute of Technology, 518000, Shenzhen, China

e-mail: gaoqingbin@gmail.com

\section{Yueying Wang}

School of Mechatronic Engineering and Automation

Shanghai University, 200444 Shanghai, China

e-mail:wyy676@126.com

Michel Zasadzinski

Research Center for Automatic Control of Nancy (CRAN)

Université de Lorraine, UMR 7039, CNRS, Cosnes-etRomain, France

e-mail: michel.zasadzinski@univ-lorraine.fr 